\title{
1 Symbiont community diversity is more constrained in holobionts \\ 2 that tolerate diverse stressors
}

3

$4 \quad$ Running title: Symbiont communities under diverse stressors

6 Lauren I. Howe-Kerr ${ }^{1}$, Benedicte Bachelot ${ }^{1}$, Rachel M. Wright ${ }^{2}$, Carly D. Kenkel ${ }^{3}$, Line K. Bay ${ }^{4}$,

7 Adrienne M.S. Correa ${ }^{1}$

$9 \quad{ }^{1}$ BioSciences at Rice, Rice University, Houston, TX 77005 U.S.A.

$10{ }^{2}$ Department of Genetics, Harvard Medical School. Boston, MA 02115 U.S.A.

$11{ }^{3}$ Department of Biological Sciences, University of Southern California, Los Angeles, CA 90089

12 U.S.A.

$13{ }^{4}$ Australian Institute of Marine Science, Townsville, Queensland, Australia

15 Corresponding author: Lauren I. Howe-Kerr, 6100 Main Street, MS-140, Houston, TX 77005,

16 Email: lih2@rice.edu, Phone: 719-252-4909, ORCID: 0000-0002-8086-5869

18 Keywords: Acropora millepora, alpha diversity, beta diversity, climate change, coral, 19 generalized joint attribute model (GJAM), Symbiodiniaceae, Vibrio owensii

21 Paper type: Primary Research 


\section{Abstract}

23 Coral reefs are experiencing global declines as climate change and other stressors cause

24 environmental conditions to exceed the physiological tolerances of host organisms and their

25 microbial symbionts (collectively termed the holobiont). To assess the role of symbiont

26 community composition in holobiont stress tolerance, diversity metrics and abundances of

27 obligate dinoflagellate endosymbionts (Family: Symbiodiniaceae) were quantified from eight

28 Acropora millepora coral colonies (hereafter called genets) that thrived under or responded

29 poorly to various stressors. Four 'best performer' coral genets were selected for analysis because

30 they survived 10 days of high temperature, high $p \mathrm{CO}_{2}$, bacterial addition, or combined stressors,

31 whereas four 'worst performer' coral genets were analyzed because they experienced significant

32 mortality under these stressors. At the end of the experimental period, seven of eight coral genets

33 mainly hosted Cladocopium symbionts, but also contained Brevolium, Durusdinium, and/or

34 Gerakladinium symbionts at lower abundances $(<0.1 \%$ of the total community). After 10 days of

35 stress, symbiont communities varied significantly among host genets, but not stress treatments,

36 based on alpha and beta diversity metrics. A generalized joint attribute model (GJAM) also

37 predicted that symbiont communities were primarily sensitive to host genet at regional scales.

38 Indicator species analysis and the regional GJAM model identified significant associations

39 among particular symbionts and host genet performance. Specifically, Cladocopium 3k

40 contributed to the success of best performer host genets under various stressful conditions,

41 whereas Durusdinium glynnii and Durusdinium trenchii were significantly associated with one

42 worst performer genet. Cladocopium 3k dominance should be more broadly investigated as a

43 potential predictor of stress resistance in Acropora millepora populations across their geographic

44 range. Symbiodiniaceae communities exhibited higher richness and variance (beta diversity) in

45 the worst performing genets. These findings highlight that symbiont community diversity metrics

46 may be important indicators of resilience in hosts central to diverse disciplines, from agriculture

47 to medicine. 


\section{Introduction}

50 Coral reefs are undergoing rapid declines in health on a global scale following increased

51 exposure to climate change stressors, such as warming sea surface temperatures and $p \mathrm{CO}_{2}$, and

52 increased loading with disease-causing agents (Hughes et al., 2017, 2018; Zaneveld et al., 2016).

53 Microbial symbionts can influence the capacity of hosts, including reef-building corals, to

54 acclimatize to environmental stressors. Dinoflagellates in the family Symbiodiniaceae reside in

55 the tissues of corals, giant clams, and other marine invertebrates, and have been empirically

56 demonstrated to influence the ability of corals to survive stress events (Baker, 2001; Baker,

57 Starger, McClanahan, \& Glynn, 2004; Kenkel \& Bay, 2018; Todd C. LaJeunesse et al., 2010;

58 Todd C. LaJeunesse, Smith, Finney, \& Oxenford, 2009; Manzello et al., 2018; Rouzé, Lecellier,

59 Saulnier, \& Berteaux-Lecellier, 2016). Particular Symbiodiniaceae species, such as

60 Durusdinium trenchii, are more likely to remain associated with hosts and/or to retain

61 photosynthetic function during acute temperature anomalies or extremes (Manzello et al., 2018;

62 Silverstein, Cunning, \& Baker, 2015; but see Silverstein et al., 2017). For example, in a

63 dominant reef-building Pacific coral (Acropora millepora), a Durusdinium species minimized

64 bleaching (a diminished host health state characterized by loss of Symbiodiniaceae en masse)

65 and increased host survival of acute thermal anomalies (Bay, Doyle, Logan, \& Berkelmans,

66 2016; Berkelmans \& van Oppen, 2006; A. M. Jones, Berkelmans, van Oppen, Mieog, \& Sinclair,

67 2008; Mieog et al., 2009). Yet, harboring these stress resistant symbionts might constitute a

68 tradeoff for corals: increased temperature tolerance at the expense of growth especially in cooler

69 environments (Cantin, van Oppen, Willis, Mieog, \& Negri, 2009; A. Jones \& Berkelmans, 2010;

70 Little, van Oppen, \& Willis, 2004). Elevated $p \mathrm{CO}_{2}$ has been shown to enhance growth and

71 photosynthetic capacity in certain Symbiodiniaceae species (Brading et al., 2011), to have no

72 effect on others (Brading et al., 2011) or to result in the loss of symbionts and their

73 photosynthetic function (Kaniewska et al., 2012). Thus, the impact of ocean acidification stress

74 on Symbiodiniaceae likely varies with taxa and context and needs further investigation.

75 Interactions between Symbiodiniaceae and bacterial pathogens (e.g., Vibrio spp.) have also

76 previously been correlated with coral (Rouzé et al., 2016) or symbiont health (Hauff et al., 2014).

77 Specifically, Acropora cytherea colonies harboring Durusdinium symbionts were more resistant

78 to infection with Vibrio spp. than conspecifics harboring Symbiodinium symbionts (Rouzé et al.,

79 2016). However, the relationship between Symbiodiniaceae identity and relative resistance to 
Vibrio infection is unexplored for most Symbiodiniaceae species, as is the relationship between symbiont community diversity metrics and host resistance to bacterial pathogens.

83 To date, most Symbiodiniaceae studies, including many of those discussed above, have focused on the contributions of individual symbiont species, rather than symbiont community composition, to holobiont stress responses. This focus invokes the 'selection effect', which assumes that the independent function of dominant species drives overall community function (Loreau et al., 2001). However, if the function of a given symbiont species differs when in the context of a diverse community (due to facilitative or other interactions - the 'complementarity effect', Fox, 2005), as compared to in monoculture, then community composition or diversity metrics may better represent symbiont contributions to holobiont physiology. For example, community diversity metrics may provide important insights in systems where low abundance symbionts contribute (even if ephemerally) to overall holobiont stress responses (e.g., LaJeunesse et al., 2009; Zaneveld, McMinds, \& Thurber, 2017, but see Lee et al., 2016). One of the first studies to investigate how symbiont attributes are associated with host performance (Kenkel \& Bay, 2018) found a decreasing trend in symbiont cooperation (in terms of autotrophically derived carbon shared with hosts) under heat stress within coral species that harbored more diverse Symbiodiniaceae communities. Another study found that coral juveniles that harbored more diverse symbiont communities exhibited lower survival than juveniles with less diverse communities (Quigley, Willis, \& Bay, 2016). There has also been a general observation that dysbiotic host individuals vary more in microbial community composition than healthy host individuals - the 'Anna Karenina Principle' or AKP (Zaneveld et al., 2017). This has

102 previously been documented in experimentally stressed coral-associated bacterial communities

103 (Zaneveld et al., 2016), but has not been examined in the context of Symbiodiniaceae

104 communities.

By identifying symbiont species occurring at abundances as low as $0.1 \%$ in a host (Quigley et 107 al., 2014), high-throughput sequencing (HTS) methods make it possible to compare symbiont 108 community characteristics associated with host genets under ambient and stressful conditions, 109 and can support future investigations into the relevance of the complementarity effect for host110 microbe symbioses. A variety of markers, including the highly variable Symbiodiniaceae

111 Internal Transcribed Spacer-2 (ITS-2) region of rDNA, have been applied in isolation or 
112

combination to assess the diversity of Symbiodiniaceae present in hosts. HTS of the Symbiodinianceae ITS-2 is useful for investigating symbiont contributions to host health and stress response at a fine scale (i.e., at the level of sequence variants, including those present at low abundances); this approach has revealed novel Symbiodiniaceae variants, host associations, and/or distribution patterns (Brener-Raffalli et al., 2018; Cunning, Gates, \& Edmunds, 2017; Green, Davies, Matz, \& Medina, 2014; Hollie M Putnam, Stat, Pochon, \& Gates, 2012; Quigley, Bay, \& Willis, 2017; Quigley et al., 2014, 2016; Quigley, Willis, \& Bay, 2017; Ziegler et al., 2017; Ziegler, Eguíluz, Duarte, \& Voolstra, 2018; Ziegler, Stone, Colman, Takacs-Vesbach, \& Shepherd, 2018). For example, variation in the abundances of background $(<10 \%$ of total community) symbionts has been documented across reefs separated by as little as $19 \mathrm{~km}$ (e.g., Green et al., 2014; van Oppen et al., 2018). It has been hypothesized that these differences in Symbiodiniaceae sequence variant distribution may be associated with fine scale environmental variation (Brener-Raffalli et al., 2018; Cunning et al., 2017; Quigley et al., 2014; Quigley, Warner, Bay, \& Willis, 2018). However, no HTS studies have examined symbiont community composition or diversity metrics (i.e., alpha and beta diversity) from host genets that perform well versus poorly under stressful conditions.

Here, we applied a HTS approach to determine whether particular Symbiodiniaceae variants (potentially species) or community characteristics were associated with host genets and/or environmental stressors. At the conclusion of experimental stress treatments, we analyzed Symbiodiniaceae communities from eight Acropora millepora genets that thrived under or responded poorly to climate stressors (high temperature and/or $p \mathrm{CO}_{2}$ ), increased exposure to a pathogenic bacteria (Vibrio owensii) in isolation, or when these stressors were combined. Coral fragment health, Symbiodiniaceae cell density, and Symbiodiniaceae identity and diversity metrics were assessed in order to identify symbiont 'species' and community diversity attributes that were associated with host survival or mortality. We hypothesized that: 1) best performing host genets will contain higher relative abundances of stress-tolerant Symbiodiniaceae (e.g., Durusinium trenchii) than worst performing genets; 2) fragments of a given host genet exposed to different stress treatments will differ significantly in their symbiont community composition and diversity, as well as from that of control fragments; and 3) worst performing host genets will have higher beta diversity than best performing ones, as predicted by the Anna Karenina Principle. 


\section{Materials and Methods}

146 Study site, experimental design, and sample collection

147 Colonies representing 8 distinct genets of Acropora millepora known to be best or worst

148 performers under stress (based on survival, Wright et al. submitted) were collected between

149 October 1-8, 2014 from three Great Barrier Reef sites (Supp. Mat. Figure 1): Pandora Island

$150 \quad\left(18^{\circ} 48^{\prime} 45^{\prime \prime} \mathrm{S}, 146^{\circ} 25^{\prime} 59.16^{\prime \prime} \mathrm{E}\right.$, Genets Worst-27, Worst-31, Worst-34), Rib Reef (18 $28^{\prime} 53.4^{\prime \prime} \mathrm{S}$,

151 146 52'24.96"E, Genets Best-12, Best-20, Best-38), and Davies Reef lagoon (18³0’3.96"S,

$152147^{\circ} 22^{\prime} 48^{\prime \prime} \mathrm{E}$, Genets Best-4, Worst-2) and transferred to flow-through seawater tanks at the

153 National Sea Simulator system at the Australian Institute of Marine Science. Each colony was

154 then split into fragments, acclimatized to a common garden condition for five months, and placed

155 into treatment conditions simulating environmental stressors (Table 1, see the Supplementary

156 Materials for details of the experimental methods).

Table 1. Overview of experimental treatments. Eight genets of Acropora millepora $(\mathrm{N}=120$

159 fragments total, with 3 replicates per genet per treatment), were exposed to control, bacteria 160 addition, elevated temperature, elevated $p \mathrm{CO}_{2}$, or combined bacteria/heat $/ p \mathrm{CO}_{2}$ stressors. After

16110 days, surviving fragments were sampled for Symbiodiniaceae density and diversity.

162

\begin{tabular}{|c|c|c|c|}
\hline Stress type & $\begin{array}{c}\text { Temperature } \\
\left({ }^{\circ} \mathbf{C}\right)\end{array}$ & $\begin{array}{c}\mathrm{pCO}_{2} \\
(\mathrm{ppm} / \mathrm{pH})\end{array}$ & $\begin{array}{c}\text { +/- Vibrio owensii } \\
6 \text { hrs at } 10^{6} \text { cells } / \mathrm{ml}\end{array}$ \\
\hline Control & 27 & $400 / 8.0$ & - \\
\hline Bacteria & 27 & $400 / 8.0$ & + \\
\hline Heat & 30 & $400 / 8.0$ & - \\
\hline$p \mathrm{CO}_{2}$ & 27 & $700 / 7.8$ & - \\
\hline Combined & 30 & $700 / 7.8$ & + \\
\hline
\end{tabular}


164

Here, we analyze the Symbiodiniaceae communities from surviving fragments $(\mathrm{N}=102)$ of four best performing (Best-4, Best-12, Best-20, Best-38) and four worst performing coral genets (Worst-2, Worst-27, Worst-31, Worst-34) following 10 days of exposure to bacteria addition, elevated temperature, elevated $p \mathrm{CO}_{2}$, or combined bacteria/heat $/ p \mathrm{CO}_{2}$ stressors (samples are a subset of those from Wright et al. submitted). Coral fragments were visually inspected and photographed with a Color Watch Card (www.coralwatch.org) every 24 hours for lesions and bleaching signs. Change in fragment color (a proxy for symbiont density and/or chlorophyll content) and buoyant weight growth rate $\left(\%\right.$ change in weight $g \cdot$ day $\left.^{-1}\right)$ were calculated from the beginning to the end of the experiment (see Supplementary Materials for details). At the end of the experiment, surviving coral fragments were snap frozen in liquid nitrogen and then stored at $80^{\circ} \mathrm{C}$ until processing.

Snap frozen coral fragments were airbrushed to remove coral holobiont tissue, and aliquots of the resulting tissue slurry were transferred to $70 \%$ ethanol for DNA extraction or fixed with 5\% formalin for Symbiodiniaceae cell density counts. Cell density samples were counted using a haemocytometer ( $\mathrm{N}=8$ replicate counts/sample), and density was calculated as cells $/ \mathrm{cm}^{2}$ by using surface area of the coral skeleton (Stimson \& Kinzie, 1991) and total volume of coral tissue blastate. Statistical differences for cell density, color change, and growth rates between treatments and genets were determined using Kruskal-Wallis and Pairwise Wilcoxon rank sum tests.

\section{Sample DNA extraction and sequencing}

DNA from each fragment was extracted using Wayne's Method (Lundgren, Vera, Peplow, Manel, \& van Oppen, 2013). To extract coral holobiont DNA, each sample was centrifuged and the ethanol was removed. The pellet was transferred to a SDS buffer and then precipitated with KOAc, followed by a series of ethanol washes. Pellets were resuspended in $30 \mu 1$ of $1 \mathrm{x}$ TAE and stored at $-20^{\circ} \mathrm{C}$. DNA concentrations were determined through Quant-iT PicoGreen dsDNA assays; 96 samples had sufficient DNA concentrations for HTS sequencing. The ITS-2 region of Symbiodiniaceae rDNA was amplified using symbiont-specific primers: SYM_VAR_5.8SII (5'GAATTGCAGAACTCCGTGAACC 3') and SYM_VAR_REV 5' (CGGGTTCWCTTGTYTGACTTCATGC 3'). The target amplicon was approximately 234266bp (Hume et al., 2018). The PCR reaction contained $5 \mu 1$ of DNA ( $5 \mathrm{ng} / \mu 1), 2.5 \mu 1$ of 
SYM_VAR_5.8SII + MiSeq Adapter (5'(5'-GTCTCGTGGGCTCGGAGATGTGTATAAGAGACAG ReadyMix, and $2.5 \mu 1$ of molecular grade water for a total reaction volume of $25 \mu 1$. PCR cycles were as follows: $95^{\circ} \mathrm{C}$ for $3 \mathrm{~min}, 15$ cycles of $95^{\circ} \mathrm{C}$ for $30 \mathrm{sec}, 56^{\circ} \mathrm{C}$ for $30 \mathrm{sec}$, and $72^{\circ} \mathrm{C}$ for 30 sec, and lastly $72^{\circ} \mathrm{C}$ for $4 \mathrm{~min}$.

PCR clean-up was completed using Agencourt AMPure XP Magnetic Beads. Illumina indexing primers were added to $50 \mu 1$ of purified PCR product, and a new PCR was run to incorporate unique barcodes for each sample. The PCR reaction contained $5 \mu 1$ of cleaned PCR product, $5 \mu 1$ of Illumina Indexed Primer 1 (i5), $5 \mu 1$ of Illumina Indexed Primer 2 (i7), $25 \mu 1$ 2x KAPA HiFi HotStart, and $10 \mu \mathrm{l}$ PCR Grade water for a total reaction volume of $50 \mu 1$. PCR cycles were as follows: $95^{\circ} \mathrm{C}$ for $3 \mathrm{~min}, 20$ cycles of $95^{\circ} \mathrm{C}$ for $30 \mathrm{sec}, 56^{\circ} \mathrm{C}$ for $30 \mathrm{sec}$, and $72^{\circ} \mathrm{C}$ for $30 \mathrm{sec}$, and lastly $72^{\circ} \mathrm{C}$ for $4 \mathrm{~min}$. The resulting PCR product was purified with Agencourt AMPure XP

212 Magnetic Beads. Samples were quantified via qPCR using the KAPA library quantification kit 213 and normalized and pooled in equal molar amounts. Pooled samples were sequenced on the

214 Illumina MiSeq platform using a PE300 run with 25\% PhiX at the Georgia Genomics and Bioinformatics Core (University of Georgia, Athens, GA).

\section{Bioinformatic Processing and Statistical Analyses}

218 Illumina's real time analysis was run during sequencing using the default settings in order to

219 remove clusters with the least reliable data. Demultiplexed fastq files were generated with Illumina's BaseSpaceFS (version 1.5.964) and reads were processed in RStudio (version 1.1.456) through the DADA2 pipeline (version 1.11.0, Callahan et al., 2016) with modifications for the Symbiodiniaceae ITS-2 region. Samples with less than 10,000 reads $(\mathrm{N}=2)$ were removed from the dataset ( $\mathrm{N}=94$ samples remained). The DADA2 pipeline generated a table of amplicon sequence variants (ASVs); alpha diversity was calculated using Shannon's Diversity $\left(H^{\prime}\right)$ index and Simpson's Diversity (1-D) index from the full ASV table. Since these data were not normally distributed based on a Shapiro-Wilk test $\left(H^{\prime}: \mathrm{W}=0.97, \mathrm{p}=0.01 ; 1-\mathrm{D}: \mathrm{W}=0.89\right.$, 
Symbiodiniaceae diversity between best and worst performers, and among treatments. A Kruskal-Wallis test was used to determine whether significant differences existed in the alpha diversity of Symbiodiniaceae ITS-2 sequence variants among treatments.

The ASV table from DADA2 was then curated via the LULU pipeline, which uses co-occurrence patterns and sequence similarity to eliminate erroneous ASVs (note that authentic singleton ASVs could also be removed, which is why alpha diversity statistics were performed prior to the LULU pipeline, Frøslev et al., 2017). Symbiodiniaceae species or ITS-2 types (hereafter, collectively referred to as species) were then assigned based on BLAST results to a local Symbiodiniaceae ITS-2 database (Cunning et al., 2017). Permutational multivariate analysis of variance using Bray-Curtis distance was used to generate an NMDS plot to visualize differences in Symbiodiniaceae community. To determine if Symbiodiniaceae community composition significantly differed between control versus treatment conditions (as a binary comparison), among treatment conditions, between best and worst performing genets, and among treatments within best and worst performing genets, we first tested for homogeneity in group (genets and treatments) dispersion using 'betadisper' in the 'vegan' R package (version 2.5.3, Oksanen et al., 2013). A significant result indicates that the groups differ significantly in beta diversity.

245 Multivariate analyses of the variance in composition are sensitive to heterogeneity, but permutational multivariate analysis of the variance ('adonis' in 'vegan') tends to be less sensitive than other tests (Anderson \& Walsh, 2013). Therefore, to test for significant differences in

248 Symbiodiniaceae composition among treatments and genets, we used the 'adonis' function in 249 'vegan'. Symbiodiniaceae species that were strongly indicative of treatment condition or genet 250 designation were examined using the Indicspecies R package (version 1.7.6, De Cáceres \& 251 Legendre, 2009). To investigate the strength of links among A. millepora genet, stress type, and

252 Symbiodiniaceae community composition at a regional (among reefs) scale, we fitted a

253 generalized joint attribute model (GJAM) in R to the LULU-curated dataset, using 10,000

254 iterations and 500 burn-in steps (Clark, Nemergut, Seyednasrollah, Turner, \& Zhang, 2017). This

255 joint probabilistic model takes into account co-dependence amongst ASVs and performs well in

256 the presence of zeros. We also used the regional GJAM model to inversely predict A. millepora 257 genet and experimental treatment given a particular Symbiodiniaceae community. Specifically, 258 for each Symbiodiniaceae sample $(y *)$, we used a Monte Carlo integration to inversely predict 259 the covariates $(x *)$ : 


$$
[x * \mid y *]=\int[x * \mid \theta, y *][\theta \mid x, y] d \theta
$$

261 where $\theta$ is the non-informative prior distribution.

263 We also estimated the Symbiodiniaceae community sensitivity to host genets and treatment.

264 After fitting the regional GJAM model, we obtained a predictors (genet and treatment)-by-

265 species matrix of coefficients $\boldsymbol{B}$ that holds all predictor-by-species responses. Elements of $\boldsymbol{B}$ are

266 the individual sensitivities of each species to each predictor. As part of the fitting process, we

267 also estimated a species-by-species covariance matrix $\Sigma$ that holds residual indirect relationships

268 between species. Sensitivity across the entire Symbiodiniaceae community is then:

$$
f=\operatorname{diag}\left(B \Sigma^{-1} B\right)
$$

271 Finally, since Rib Reef genets were all best performers and Pandora Island genets were all worst

272 performers, additional GJAM models were fitted to genets originating from these locations,

273 respectively, to test for treatment effects on more local scales. These local models were identical 274 to the regional GJAM model except that they were fitted only with the data from Pandora Island 275 or Rib Reef.

\section{Results}

\section{Response to experimental treatments}

279 In response to 10 days of exposure to single or combined stressors, the most coral mortality was 280 observed in the $p \mathrm{CO}_{2}$ and combined stressors treatments $(\mathrm{N}=5$ fragments total per treatment, 281 represented as dark grey bars in Figure 1); none of the control fragments experienced mortality. 

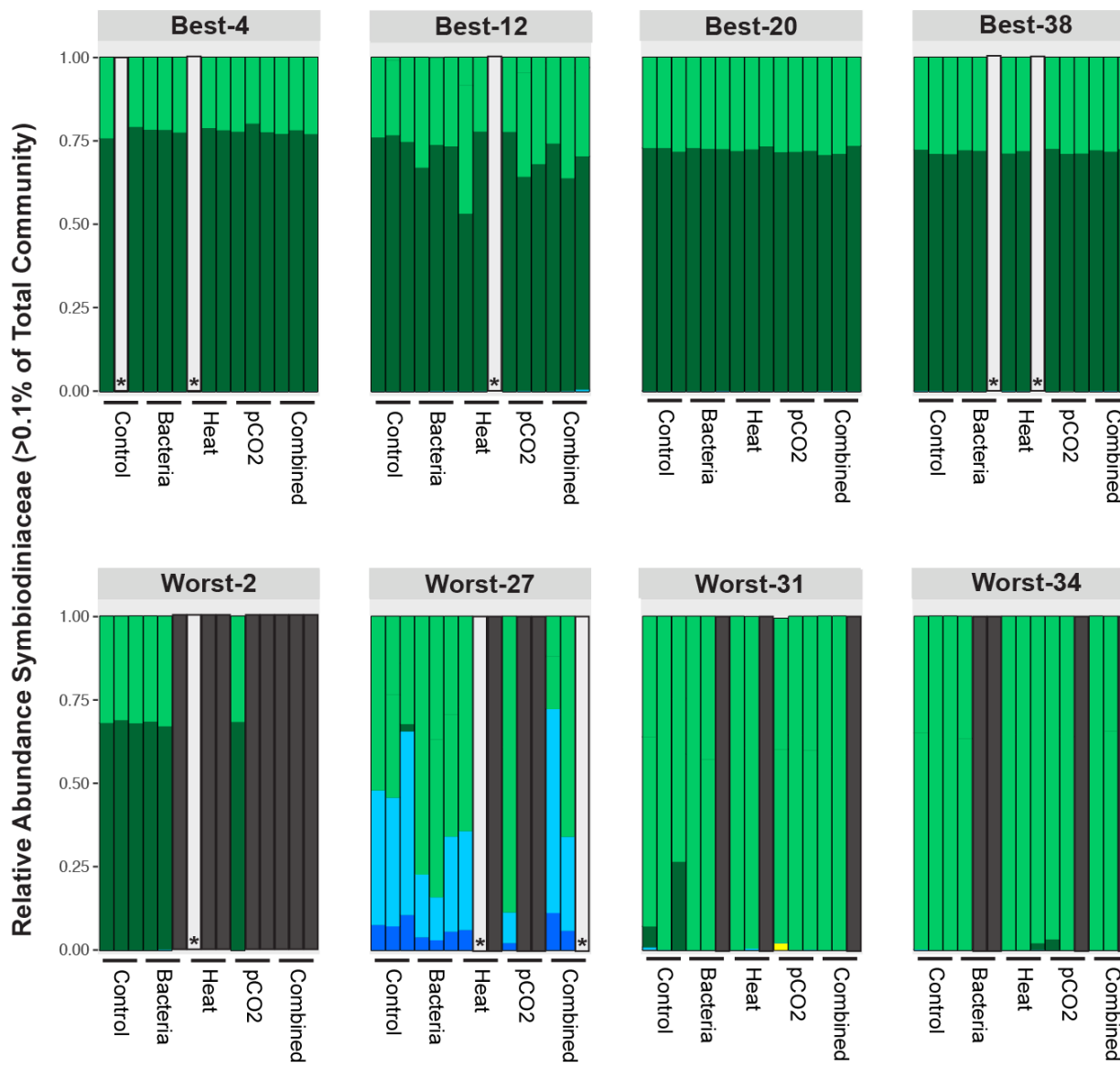

Symbiodiniaceae species Cladocopium 1232 Cladocopium $3 \mathrm{k}$ Durusdinium glynnii Durusdinium trenchii Gerakladium 3.1
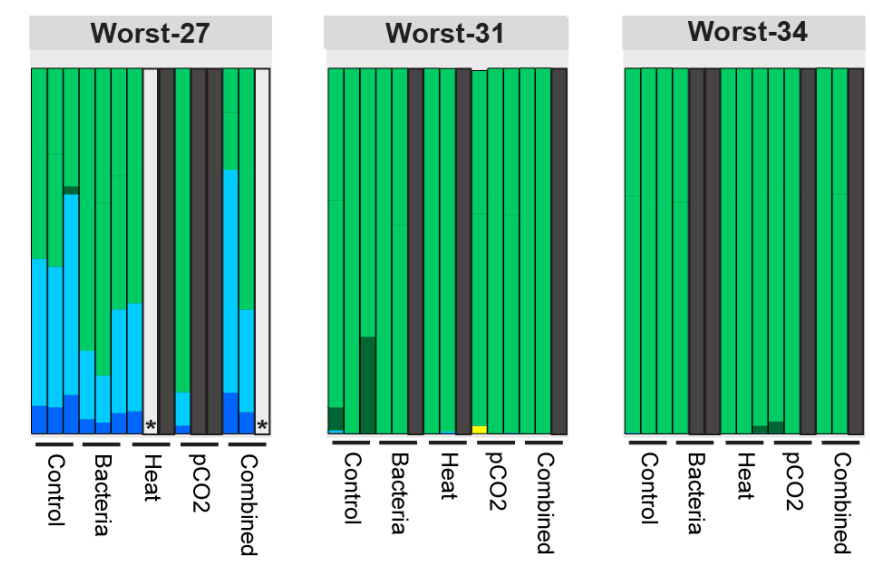

Dead

* Low DNA yield or \# reads recovered

Figure 1. Relative abundance (comprising $>0.1 \%$ of total community) of Symbiodiniaceae species or Internal Transcribed Spacer-2 (ITS-2) types in individual fragments (vertical bars) of Acropora millepora genets exposed to various experimental stressors.

Symbiodiniaceae cell density counts at the end of the experiment ranged from $1.8 \mathrm{e}^{6}$ to $1.6 \mathrm{e}^{4}$ cells $/ \mathrm{cm}^{2}$ (average for best performers: $3.5 \mathrm{e}^{5} \pm 2.1 \mathrm{e}^{5}$ cells $/ \mathrm{cm}^{2}$; average for all worst performers: $4.1 \mathrm{e}^{5} \pm 4.0 \mathrm{e}^{5}$ cells $/ \mathrm{cm}^{2}$; Figure 2). Worst-34 fragments had the highest average Symbiodiniaceae cell densities $\left(7.9 \mathrm{e}^{5} \pm 5.0 \mathrm{e}^{5}\right.$ cells $/ \mathrm{cm}^{2}$, Figure 2$)$, inflating the average number of cell density for the worst performers (average for worst performers without Worst-34: $2.3 \mathrm{e}^{5} \pm 1.6 \mathrm{e}^{5} \mathrm{cells} / \mathrm{cm}^{2}$ ). Worst-2 and Worst-31 had significantly lower Symbiodiniaceae cell densities at the end of the experiment than other coral genets (Pairwise Wilcoxon rank sum test, Worst-2 lower than Worst27 and Best-4, $\mathrm{p}=0.032$; Worst-31 lower than Best-4, Best-12 and Worst-27, $\mathrm{p}=0.018$, Supp. Mat. Table 1). Symbiont cell density was not significantly different among treatments (KruskalWallis chi-squared $=3.40, \mathrm{df}=4, \mathrm{p}=0.49$ ), but based on fragment color change from the beginning to the end of the experiment, the heat treatment depressed Symbiodiniaceae traits significantly 
more than the combined stressor treatment (Pairwise Wilcoxon rank sum test, Heat lower than combined stressors, $\mathrm{p}=0.02$ ). Buoyant growth rates were not significantly different among treatment conditions for genets that were dominated by similar symbiont communities (Best-4, Best-12, Best-20, Best-38, Worst-2 in Figure 1, Supp. Mat. Table 2).

303

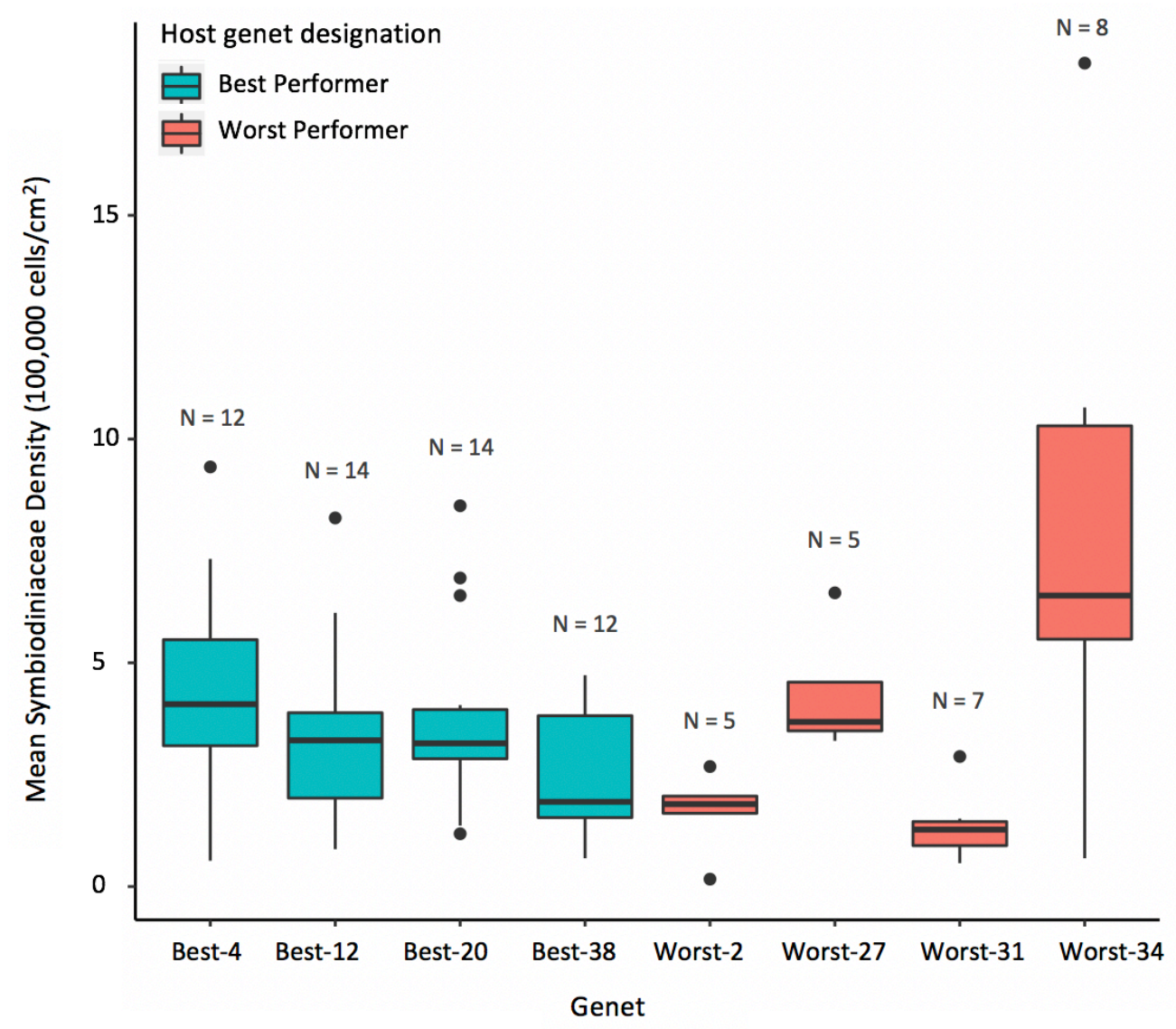

Figure 2. Average Symbiodiniaceae cell density counts from Acropora millepora genets sampled at the end of $\mathbf{1 0}$ days of experimental stress treatments. Sample sizes are provided above each genet. Worst-2 and Worst-31 had significantly lower Symbiodiniaceae cell density counts than other A. millepora genets (Worst-2 lower than Worst-27 and Best-4; Worst-31 lower than Best-4, Best-12, Best-20 and Worst-27; Supp. Mat. Table 1).

312 Of the 102 fragments that survived the experimental conditions, 96 yielded sufficient DNA for 313 amplicon sequencing (Figure 1). A total of 29,466,474 raw sequence reads were recovered from 314 these samples. After processing through the DADA2 pipeline, paired reads per sample ranged 
315 from 589,944 to 996. Two samples that contained $<10,000$ reads (one Best-4 Control and one 316 Best-4 Heat sample) were removed, leaving a total of 9,984,782 paired reads (Supp. Mat. Table 317 3) from which 232 ASVs were identified. The LULU pipeline resolved 12 ASVs from this 318 dataset. One of these ASVs (represented by 12 reads total among two samples) was discarded 319 because it did not produce any sequence similarities during the taxonomic assignment process. 320 Some of the remaining 11 ASVs were subsequently assigned to the same Symbiodiniaceae 321 species. Species assignment post-LULU ultimately identified a total of seven Symbiodiniaceae 322 species, representing four genera, from the dataset: Breviolum minutum, Cladocopium 1232, 323 Cladocopium 3k, Durusdinium glynnii, Durusdinium trenchii, Durusdinium 2, and Gerakladium

3243.1 (Table 2). Fragments of Worst-31 collectively harbored the most Symbiodiniaceae diversity 325 (all seven species), whereas fragments of Worst-2 collectively harbored the fewest (three)

326 Symbiodiniaceae species (Table 2). Most individual coral fragments (94\%, N = 88 of 94, Supp.

327 Mat. Table 4) analyzed in this study harbored more than one Symbiodiniaceae species (see Table 3282 for all detected species, Figure 1 for species comprising $>0.1 \%$ of total Symbiodiniaceae 329 community). Fragments in the $\mathrm{CO}_{2}$ treatment collectively harbored the most (all seven) 330 Symbiodiniaceae species, whereas fragments in the combined stressor treatment (bacterial 331 addition, elevated temperature and $p \mathrm{CO}_{2}$ ) harbored the fewest (four) Symbiodiniaceae species 332 (Table 2). 
Table 2. Symbiodiniaceae amplicon sequence reads analyzed and Symbiodiniaceae species (or Internal Transcribed Spacer-2 region types) resolved from Acropora millepora fragments across several experimental stress treatments. The 'Symbiodiniaceae species' column lists the species or ITS-2 types cumulatively detected across all fragments of a host genet. C3k = Cladocopium 3k (NCBI Accession \#: AY589737), C1232= Cladocopium 1232 (Accession \#: EU118163.1), D2 = Durusdinium 2 (Accession \#: AY686649), and G3.1 = Gerakladium 3.1 (Accession \#: KC597688).

\begin{tabular}{|l|l|l|}
\hline $\begin{array}{l}\text { Host genet or } \\
\text { treatment }\end{array}$ & \# Reads & \multicolumn{1}{|c|}{ Symbiodiniaceae species } \\
\hline Best-4 & $1,586,758$ & Breviolum minutum, C3k, C1232, D. glynnii, Durusdinium trenchii \\
\hline Best-12 & $1,198,509$ & B. minutum, C3k, C1232, D. glynnii \\
\hline Best-20 & $2,293,707$ & C3k, C1232, D. glynnii, D. trenchii, D2 \\
\hline Best-38 & $1,508,277$ & C3k, C1232, Durusdinium glynnii, G3.1 \\
\hline Worst-2 & 639,548 & C3k, C1232, D. glynnii \\
\hline Worst-27 & 673,979 & C3k, C1232, D. glynnii, D. trenchii \\
\hline Worst-31 & 766,193 & B. minutum, C3k, C1232, D. glynnii, D. trenchii, D2, G3.1 \\
\hline Worst-34 & $1,317,811$ & B. minutum, C3k, C1232, D. glynnii \\
\hline Control & $2,288,947$ & B. minutum, C3k, C1232, D. glynnii, D. trenchii, G3.1 \\
\hline Bacteria & $1,764,362$ & B. minutum, C3k, C1232, D. glynnii, D. trenchii \\
\hline Heat & $1,900,830$ & B. minutum, C3k, C1232, D. glynnii, D. trenchii, D2 \\
\hline pCO2 & $2,066,054$ & B. minutum, C3k, C1232, D. glynnii, D. trenchii, D2, G3.1 \\
\hline Combined & $1,964,589$ & C3k, C1232, D. glynnii, D. trenchii \\
\hline
\end{tabular}

344 Based on all ASVs identified by DADA2 (prior to processing the dataset through LULU), 345 Symbiodiniaceae alpha diversity varied categorically by genet performance but did not differ 346 based on treatment (Figure 3). Symbiont communities of worst performers were significantly 347 more diverse by Shannon's Diversity (H') Index estimates (worst performer genets: 1.62, best 348 performer genets: 1.20 , Wilcoxon rank sum test: $\mathrm{W}=2083, \mathrm{p}<0.001)$ and Simpson's $(1-\mathrm{D})$ 
Diversity estimates (worst performer genets: 0.68, best performer genets: 0.45 , Wilcoxon rank sum test, $\mathrm{W}=2118, \mathrm{p}<0.001)$. Shannon's Diversity $\left(\mathrm{H}^{\prime}\right)$ Index (Kruskal-Wallis chi-squared $=$ 1.7423, $\mathrm{df}=4, \mathrm{p}=0.78)$ and Simpson's (1-D) Diversity results (Kruskal-Wallis chi-squared = was the most diverse genet $\left(\mathrm{H}^{\prime}=1.84 ; 1-\mathrm{D}=0.76\right)$, whereas A. millepora Best-4 was the least diverse genet $\left(\mathrm{H}^{\prime}=1.04 ; 1-\mathrm{D}=0.38\right.$; Figure 3$)$.
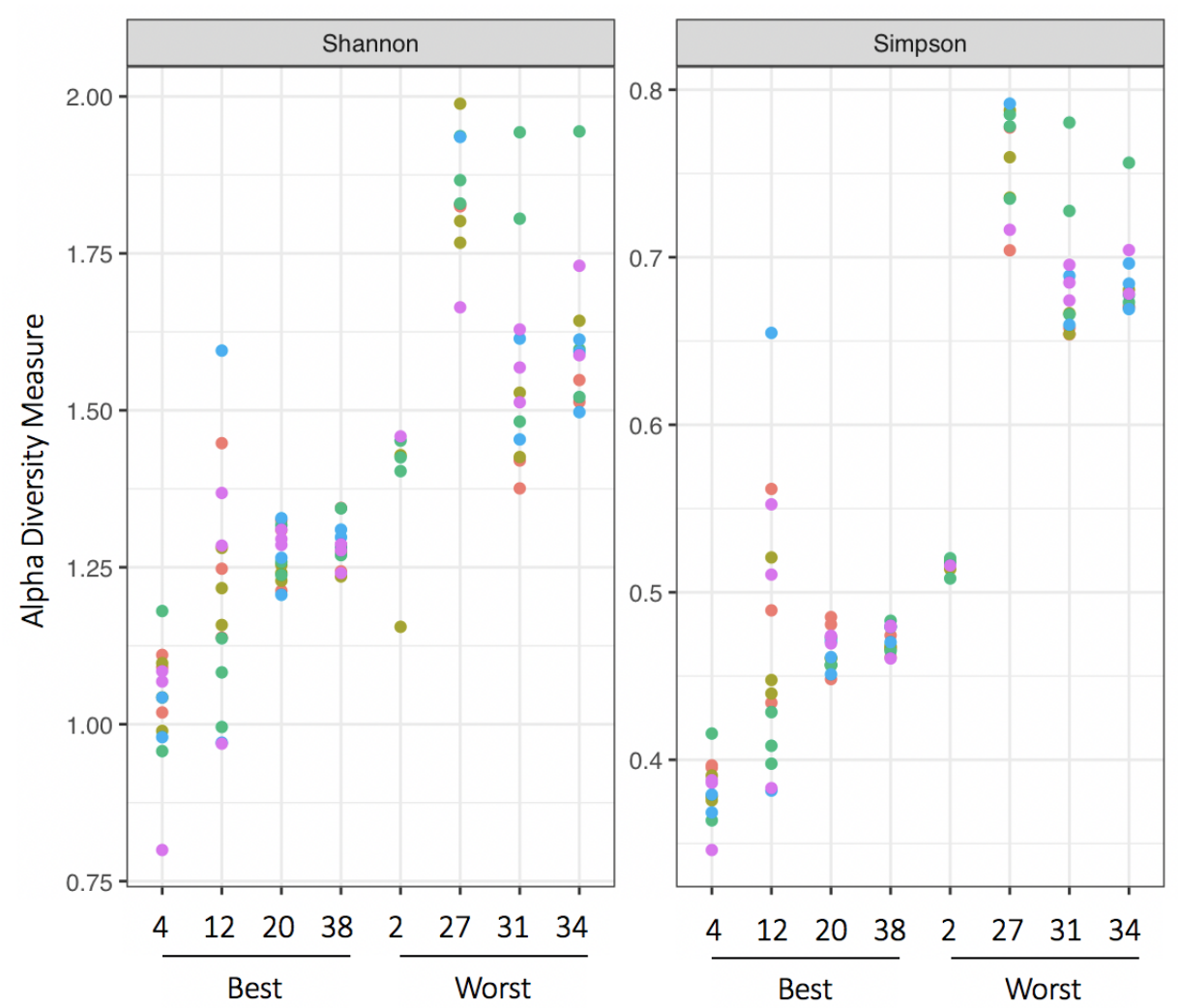

Treatment

- Control

- Bacteria

- Heat

- $\mathrm{PCO}_{2}$

- Combined

Figure 3. Alpha diversity of Symbiodiniaceae Internal Transcribed Spacer-2 (ITS-2) amplicon sequence variants by Acropora millepora genet and treatment. Shannon's Diversity Index results are reported as H', Simpson's Diversity Index results are reported as 1-D. Alpha diversity indices were calculated from the full untrimmed dataset after processing by DADA2.

A permutation test for homogeneity of multivariate dispersions also indicated that variances, a proxy for beta diversity, were significantly lower among Symbiodininaceae communities associated with best performing A. millepora genets, than among communities associated with worst performing genets $(\mathrm{df}=1, \mathrm{p}<0.001$, Figure 4$)$. In contrast, the same test showed no 
366 differences among the variances of Symbiodiniaceae communities exposed to stress versus

367 control conditions (as a binary comparison, $\mathrm{df}=1, \mathrm{p}=0.84$ ), control conditions versus single

368 stressors versus combined stressors $(\mathrm{df}=2, \mathrm{p}=0.98)$, or among all of the different stress treatments

369 (including combined stressors, $\mathrm{df}=4, \mathrm{p}=0.96$ ). Permutational multivariate analysis of variance

370 supported that Symbiodiniaceae communities differed significantly between best versus worst

371 performing $A$. millepora genets (Adonis with Bray-Curtis distance, $\mathrm{p}<0.001$, Figure 4 ). In

372 contrast, Symbiodiniaceae communities among treatment conditions were not significantly

373 different (Adonis with Bray-Curtis distance, $p=0.99$ ). Within best or worst performing coral

374 genets (as groups), stress treatment was also not associated with significantly different symbiont

375 assemblages (Adonis with Bray-Curtis distance, Best performers, $p=0.75$; Worst performers, $p$

$376=0.81)$. 


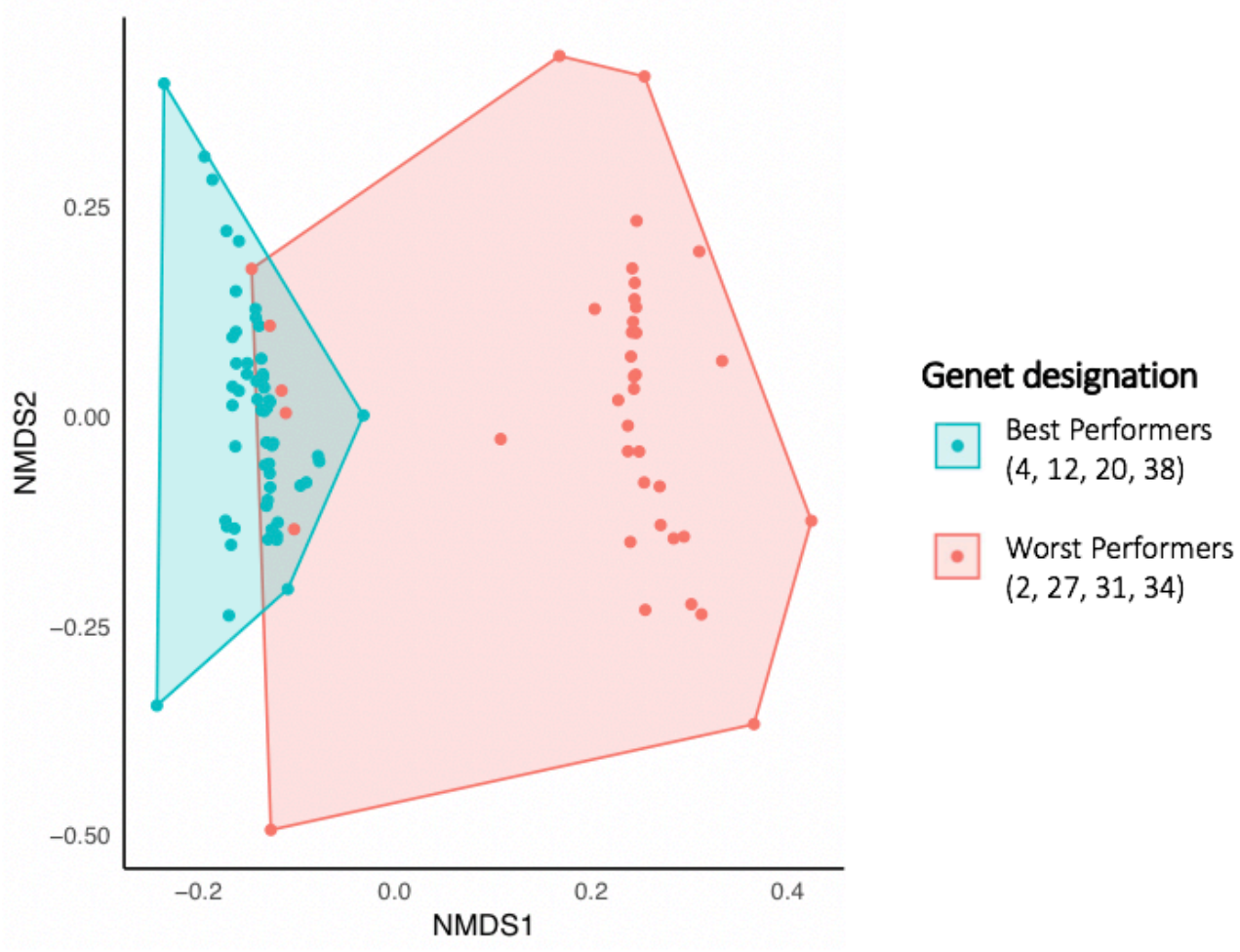

Figure 4. Symbiodiniaceae communities associated with Acropora millepora best performer genets differ from those associated with worst performer genets, based on non-metric multidimensional scaling (NMDS) using a Bray Curtis distance matrix. Variances, a proxy for beta diversity, are significantly higher among Symbiodininaceae communities associated with worst performer $A$. millepora genets, than among communities associated with best performer genets. The six Symbiodiniaceae communities with the lowest scores on NMDS1 represent the Worst-2 genet, collected from Davies Reef (along with Best-4).

When all three reef locations were incorporated into the regional GJAM model, it fitted the data well (Supp. Mat. Figure 2) and indicated that Symbiodiniaceae communities were sensitive to genet, but not to treatment (Figure 5 and sensitivity analysis shown in Supp. Mat. Figure 3). Using this regional model, we inversely predicted the A. millepora genet and experimental treatment given a particular Symbiodiniaceae community. Based on this process, some $A$. millepora genets were easily predicted; they had distinctive Symbiodiniaceae communities associated with them. This includes Best-4, Best-12, and Best-20, as well as Worst-2 (Figure 5). In contrast, prediction of experimental treatments from Symbiodiniaceae communities was not 
robust using the regional model (Figure 5). However, Symbiodiniaceae community was more sensitive to treatment in local GJAM models, which were run for Symbiodiniaceae communities at Pandora Reef and Rib Reef, respectively (Supp. Mat. Figure 3 compared to Supp. Mat. Table sensitivities for every stress type except bacterial addition and elevated temperature (Supp. Mat.

402 Table 5).

403

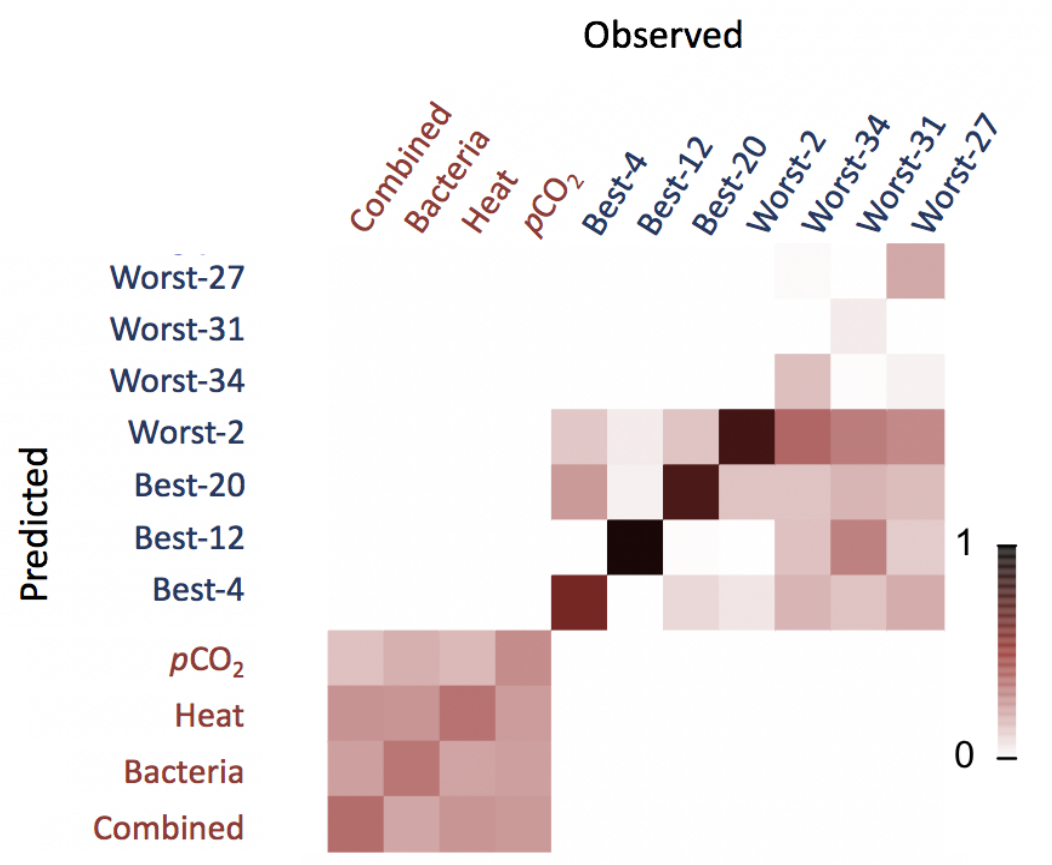

404

Figure 5. GJAM inverse predictions of Acropora millepora genet and experimental treatment based on Symbiodiniaceae community composition. Darker colors indicate that the A. millepora genet or treatment condition was more readily predicted from the Symbiodiniaceae community via the regional GJAM model.

Symbiodiniaceae characteristics in best and worst performing coral holobionts

411 The dominant Symbiodiniaceae species in best performer A. millepora genets were more similar

412 to each other than the dominant species associated with worst performer genet (Figure 1).

413 Certain Symbiodiniaceae species were significantly associated with genet performance, or a

414 stress treatment. Based on the indicspecies R package, Cladocopium 3k was a significant

415 indicator species for best performer genets $(\mathrm{p}=0.005)$. Cladocopium $3 \mathrm{k}$ was also present at

416 similar relative abundances within Worst-2 fragments, but this worst performer genet contained 
417 fewer background symbionts relative to best performer host genets (Table 2). Cladocopium 418 1232, Durusdinium trenchii, and Durusdinium 1 were indicator species for worst performer 419 genets ( $\mathrm{p}=0.005$ for each). Gerakladium 3.1 was a significant indicator species for the $p \mathrm{CO}_{2}$

420 treatment $(\mathrm{p}=0.03)$ but only represented $>1 \%$ of the total Symbiodiniaceae in one coral fragment

421 in the study; there were no significant indicator species for the other treatments.

423 Results of the regional GJAM model indicated similar significant associations of

424 Symbiodiniaceae species with certain coral genets (Figure 6). Cladocopium 3k_1 was strongly

425 associated with Best-38 (labeled 'intercept' in Figure 6) and strongly disassociated with Worst-

426 27, Worst-31, and Worst-34. Cladocopium 1232_1 was also strongly associated with Best-38,

427 whereas Cladocopium 1232_2 and Cladocopium 3k_2 were strongly disassociated with that

428 genet. Cladocopium 1232_3 was also strongly disassociated with Best-38 and strongly associated

429 with Worst-27, Worst-31, and Worst-34 and Best-12. Durusdinium trenchii and Durusdinium 1

430 were strongly associated only with Worst-27 (Figure 6). 


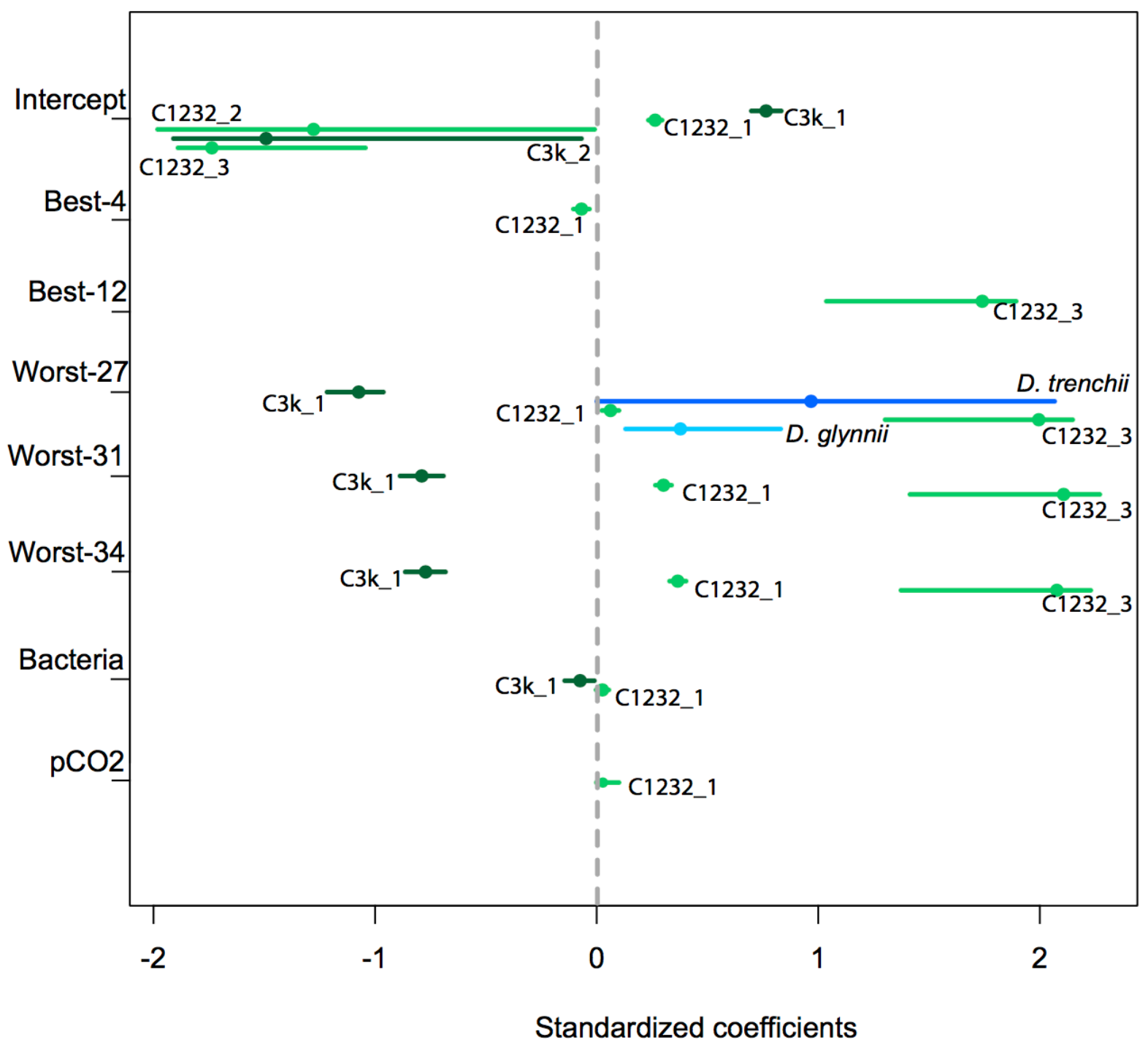

433 Figure 6. Posterior distributions of the significant effects of treatment and coral genet. Dots

434 indicate the median and the segments expand to the $95 \%$ credible intervals. Light green $=$

435 Cladocopium 3k ASVs; dark green =Cladocopium 1232 ASVs; light blue = Durusdinium

436 glynnii ASVs; and dark blue = Durusdinium trenchii ASVs.

\section{Discussion}

439 Specific symbionts are associated with host survival under diverse stressors

440 This study demonstrates that different specific symbiont species are differentially associated with

441 A. millepora host genets that exhibited high or low survivorship when challenged by various

442 stressors. Both an indicator species analysis and a regional generalized joint attribute model

443 (GJAM), identified a significant association between Cladocopium 3k and best performer 
444 genet(s) (Figure 6). Similar to this finding, Manzello et al., (2018) reported that $73 \%$ of the

445 distribution of Durusdinium trenchii in a dominant reef-building Caribbean coral (Orbicella

446 faveolata) was attributable to host identity. GJAM, which takes into account co-dependence

447 among Symbiodininaceae species, also found a strong disassociation between Cladocopium 3k_1

448 and three of the four worst performer genets (Figure 6). Cladocopium 3k has previously been

449 reported from a diversity of Acropora species (Barshis, Ladner, Oliver, \& Palumbi, 2014; Smith,

450 Pinzón, \& LaJeunesse, 2009), including A. millepora (LaJeunesse et al., 2004). Most recently,

451 Cladocopium 3k was reported from diverse host genera at mesophotic depths (Echinophyllia,

452 Fungia, Pachyseris, and Pavona from 45-70m, Bongaerts et al., 2011) suggesting that this

453 symbiont can cope with (or has locally adapted to) diverse light and temperature profiles.

454 However, Cladocopium $3 k$ has not previously been linked to stress tolerance; it is more often

455 predicted to be thermally susceptible, for example, relative to Durusdinium 2 in American

456 Samoan acroporids (e.g., Barshis et al., 2014). Based on recent coral transcriptomic studies, we

457 infer that Cladocopium 3k may have contributed to the success of best performer host genets

458 through differences in its baseline expression of various genes (relative to other Symbiodiniaceae

459 species examined in this study), rather than transcriptional responses to stressors (Barshis et al.,

460 2014; Leggat et al., 2011; H. M. Putnam, Mayfield, Fan, Chen, \& Gates, 2013 but see

461 Baumgarten et al., 2013). The physiology of Cladocopium 3k should be further characterized in

462 monoculture and polyculture, as well as in the diverse Pacific Acropora coral species that host it.

463 Beyond insights into symbiont physiology and function, such research efforts can yield insights

464 into whether selection or complementarity effects are important in predicting and managing for

465 holobiont stress tolerance.

467 We hypothesized that best performing host genets would contain higher relative abundances of 468 stress-tolerant Symbiodiniaceae (e.g., Durusinium trenchii) than worst performing genets.

469 Contrary to this, Durusdinium glynnii and Durusdinium trenchii, as well as Cladocopium 1232 ,

470 were largely indicator species for worst performer genets based on indicator species analysis and

471 GJAM. In Worst-27, D. glynnii and D. trenchii represented from 72 to $11 \%$ of the total

472 Symbiodiniaceae community (Figure 1, Supp. Mat. Table 4). In all other coral fragments

473 analyzed in this study, Durusdinium represented $<1 \%$ of the total Symbiodiniaceae community

474 (Supp. Mat. Table 4). Although some Durusdinium species have previously been associated with

475 holobiont thermotolerance (e.g., the ITS-1 D in Berkelmans \& van Oppen, 2006; Jones et al., 
2008), Durusdinium trenchii is not necessarily tolerant of variable thermal conditions (Howells,

477 Berkelmans, van Oppen, Willis, \& Bay, 2013). Given the mortality suffered by Worst-27, there

478 is no indication that Durusdinium species provided their host corals with tolerance to elevated

479 temperature, $p \mathrm{CO}_{2}$, additions of Vibrio owensii, or combinations thereof in this experiment (see

480 mortality and relative abundance of Durusdinium species in Figure 1). The significant

481 associations among symbiont species and host genets, but not treatments, at the regional scale

482 (Figure 5) suggests that Symbiodininaceae communities might be more strongly structured by

483 the host (or biotic interactions) in A. millepora, rather than imposed by the environment. Yet,

484 within a reef, where there is less variation in host genet, some influence of environment (i.e.,

485 treatment) on symbiont community composition becomes apparent (Supp. Mat. Table 5). This

486 fits the general expectation that history and evolutionary processes, in this case contributing to a

487 heterogeneous distribution of host genets, might have a greater impact at regional (among reefs)

488 as opposed to local (within reef) scales (e.g., Witman, Etter, \& Smith, 2004).

Host genet, but not stress type, is predictive of symbiont community attributes among reefs

491 Beyond individual Symbiodiniaceae species, symbiont community attributes (alpha and beta

492 diversity) also differed significantly between best versus worst performer coral genets, but not

493 stress type. Best performer host genets had fewer Symbiodiniaceae ASVs and higher community

494 evenness than worst performer host genets (Figure 3). Symbiont communities among best

495 performer coral fragments were similar to each other, whereas symbiont communities among

496 fragments of worst performer coral genets exhibited high variance or beta diversity (Figure 4).

497 These findings agree with a recent general observation that dysbiotic host individuals vary more

498 in microbial community composition than healthy host individuals - the 'Anna Karenina

499 Principle' or AKP (Zaneveld et al., 2017). This has previously been documented in

500 experimentally stressed coral-associated bacterial communities (Zaneveld et al., 2016) and in the

501 survival of coral juveniles from distinct parental genets (Quigley et al., 2016). Inverse

502 predictions of host genet from Symbiodiniaceae community composition by the regional GJAM

503 model support this: the model easily predicted most of the best performer coral genets (Best-4,

504 Best-12, Best-20 in Figure 5), but could only reliably predict one worst performer genet (Worst-

505 2). It should be noted, however, that coral fragments exposed to stress treatments did not exhibit

506 higher variance in symbiont communities compared to control fragments at the end of the

507 experiment. Thus, our data fit with the AKP in that healthy (best performer) coral genets host 
more similar Symbiodiniaceae communities, but our data do not represent the AKP in terms of exhibiting a “constrained 'core' of control microbiomes surrounded by a large 'halo' or 'smear' of stressed or diseased microbiomes", as described by Zaneveld et al., (2017). This is the first study to directly assess whether the AKP is applicable to Symbiodiniaceae communities. These symbionts join a growing list of microbial communities, particularly those associated with various organ systems in primates (Chen et al., 2015; Halfvarson et al., 2017; Moeller et al.,

514 2013; Wu et al., 2016) that exhibit stochastic responses to environmental stressors. Future HTS 515 studies examining Symbiodiniaceae diversity from a whole community perspective can further 516 clarify the relationship between microbiome stability and host performance as reefs and other 517 systems continue to be challenged with diverse stressors.

\section{Absence of tradeoffs in symbiont community under challenge by diverse stressors}

520 Fragments of best performer coral genets were dominated by Cladocopium 3k (with a lower relative abundance of Cladocopium 1232) regardless of the stress treatment that fragments experienced in this study (Figure 1). Symbiodiniaceae community composition also did not change significantly among treatments within a given worst performer genet (Figure 1). Thus,

524 we reject our hypothesis that fragments of a given host genet exposed to different stress 525 treatments would differ significantly in their symbiont community composition and diversity, as well as from that of control fragments. This is consistent with recent findings that bacterial communities of Acropora tenuis are highly specific to host genet regardless of challenge with various environmental stressors (Glasl, Smith, Bourne, \& Webster, 2019). Furthermore, Symbiodiniaceae communities were minimally different in fragments experiencing multiple stressors, as compared to one stressor (Figure 1), although the total diversity of Symbiodiniaceae species (including those present at $<1 \%$ of the total community) was reduced in fragments experiencing combined stressors (Table 2).

534 Tradeoffs might have been expected in the growth rates of hosts harboring different

535 Symbiodiniaceae communities under control versus stress treatments. For example, it has 536 previously been documented that corals dominated by some symbionts in Durusdinium exhibit

537 higher thermotolerance, but slower growth rates, than the same host species dominated by some 538 symbionts in Cladocopium (Jones \& Berkelmans, 2010; Little et al., 2004). In this study, 539 symbiont community compositions were determined post-hoc and unfortunately, sample sizes 
540 proved insufficient to test for tradeoffs among distinct symbiont communities. However, five

541 host genets (Best-4, Best-12, Best-20, Best-38 and Worst-2) contained a particular symbiont

542 community ( 70\% Cladocopium 3k and nearly 30\% Cladocopium $1232+$ background

543 symbionts, Figure 1, Table 2). Across these fragments dominated by Cladocopium 3k (and to a

544 lesser extent, 1232), there was no apparent change in host growth rate under control versus stress

545 conditions, or among different stressors, based on log growth of fragments over the 10-day

546 duration of this study (Supp. Mat. Table 2). The lack of detectable tradeoffs in hosting the same

547 Symbiodiniaceae community under different stressors found in this study agrees with findings by

548 Wright et al. (submitted) for host genets. Based on measurements of seven host-specific traits

549 (e.g., host chromoprotein content, instant calcification rate, buoyant weight increase) from the

550 same coral fragments (as well as 30 additional host genets), host genets that performed well

551 under one stress tended to survive other stressors as well (Wright et al. submitted). Thus, our

552 findings related to symbiont communities are not likely to be mere artifacts of the eight host 553 genets examined here.

\section{Methodological considerations}

556 This study compares Symbiodiniaceae communities in fragments of individual coral genets that

557 experienced stress treatments for 10 days to fragments that experienced ambient conditions

558 during the experimental period. Data on symbiont community composition at T0 are not

559 available and therefore we cannot directly assess the extent to which symbiont communities were

560 heterogeneously distributed in colonies prior to fragmenting for the experiment, or whether stress

561 treatments caused symbiont communities to shift within individual coral fragments. Specifically,

562 Cladocopium $3 k$ could have been lost from stress-treated fragments of Worst-31 over the course

563 of the experiment, given the symbiont community composition in control Worst-31 fragments at

564 the final time point (Figure 1). However, the dominant symbiont species present in the three

565 control fragments of each coral genet at the final time point were remarkably similar (Figure 1)

566 suggesting that intracolony spatial heterogeneity in symbiont distributions and symbiont

567 shuffling have minimal explanatory power (if any) in this dataset.

568

569 In this study, genets were generally assessed in terms of their performance, yet our

570 characterization of the symbiont community compositions and cell densities of worst performer

571 genets was based on surviving fragments only. We cannot examine post-hoc whether individual 
572 worst performer fragments that experienced mortality had unique symbiont community

573 characteristics (relative to surviving worst performer fragments of the same genet) that ultimately

574 contributed to fragment death. Quantification of symbiont density per host genet in this study is

575 also based on surviving fragments only. Many worst performer coral fragments bleached as they

576 died, but these fragments were not represented in average symbiont density calculations for

577 worst performer genets. Thus, the extent to which stressors negatively impacted worst performer

578 genet health, and the difference in bleaching that occurred between worst versus best performer

579 host genets, is likely underestimated in this study.

This study is unique in that symbiont community composition was examined from host genets

582 that were known best or worst performers, based on their survival under various stressful

583 conditions. Importantly, we found that symbiont community composition and diversity metrics

584 were highly similar among best performer host genets, whereas symbiont compositions were

585 more 'dysbiotic' or variable in worst performer genets. Indicator species analysis and the

586 regional GJAM model also highlighted several Symbiodiniaceae species that were strongly

587 associated with best or worst performer genets. The presence of a similar symbiont community

588 across diverse stressors in best performer host genets provides (limited) hope for coral reefs: up

589 to a point, best performer host-symbiont combinations can potentially resist diverse or multiple

590 stressors. The extent to which we can identify and promote specific symbionts (e.g.,

591 Cladocopium 3k), communities or their characteristics (e.g., target levels of alpha, beta diversity)

592 across diverse host genets and species is a logical next goal in managing for coral reef resistance

593 and resilience.

\section{Acknowledgments}

596 We thank Noah Workman at Georgia Genomics Facility for consultation regarding Illumina 597 sequencing and Avery Zaleski for assistance processing sequence data. This study was made 598 possible through support from the U.S. National Science Foundation (OCE \#1635798 to AMSC), 599 a Sigma Xi Grant-in-Aid of Research to LHK, and an AIMS funding to LKB. Samples were 600 collected under general AIMS permit G11/34671.1 and G14/37318.1. The authors would like to 601 acknowledge experimental assistance by Maria Nayfa and Ari Muskat and have no conflicts of 602 interest to report. 
604

605

606

607

608

609

610

611

612

613

614

615

616

617

618

619

620

621

622

623

624

625

626

627

628

629

630

631

632

633

\section{References}

Anderson, M. J., \& Walsh, D. C. I. (2013). PERMANOVA, ANOSIM, and the Mantel test in the face of heterogeneous dispersions: What null hypothesis are you testing? Ecological Monographs, 83(4), 557-574. https://doi.org/10.1890/12-2010.1

Baker, A. C. (2001). Reef corals bleach to survive change. Nature, 411, 765-766. https://doi.org/10.1038/35081151

Baker, A. C., Starger, C. J., McClanahan, T. R., \& Glynn, P. W. (2004). Corals' adaptive response to climate change. Nature, 430, 741. https://doi.org/10.1038/430741a

Barshis, D. J., Ladner, J. T., Oliver, T. A., \& Palumbi, S. R. (2014). Lineage-specific transcriptional profiles of Symbiodinium spp. unaltered by heat stress in a coral host. Molecular Biology and Evolution, 31(6), 1343-1352. https://doi.org/10.1093/molbev/msu107

Baumgarten, S., Bayer, T., Aranda, M., Liew, Y. J., Carr, A., Micklem, G., \& Voolstra, C. R. (2013). Integrating microRNA and mRNA expression profiling in Symbiodinium microadriaticum, a dinoflagellate symbiont of reef-building corals. BMC Genomics, 14, 704. https://doi.org/10.1186/1471-2164-14-704

Bay, L. K., Doyle, J., Logan, M., \& Berkelmans, R. (2016). Recovery from bleaching is mediated by threshold densities of background thermo-tolerant symbiont types in a reefbuilding coral. Royal Society Open Science, 3, 160322. https://doi.org/10.1098/rsos.160322

Berkelmans, R., \& van Oppen, M. J. H. (2006). The role of zooxanthellae in the thermal tolerance of corals: A "nugget of hope" for coral reefs in an era of climate change. Proceedings of the Royal Society B: Biological Sciences, 273, 2305-2312. https://doi.org/10.1098/rspb.2006.3567

Bongaerts, P., Sampayo, E. M., Bridge, T. C. L., Ridgway, T., Vermeulen, F., Englebert, N., ... Hoegh-Guldberg, O. (2011). Symbiodinium diversity in mesophotic coral communities on the Great Barrier Reef: a first assessment. Marine Ecology Progress Series, 439, 117-126. https://doi.org/10.3354/meps09315

Brading, P., Warner, M. E., Davey, P., Smith, D. J., Achterberg, E. P., \& Suggett, D. J. (2011). Differential effects of ocean acidification on growth and photosynthesis among phylotypes of Symbiodinium (Dinophyceae). Limnology and Oceanography, 56(3), 927-938. 
Brener-Raffalli, K., Clerissi, C., Vidal-Dupiol, J., Adjeroud, M., Bonhomme, F., Pratlong, M., ... Toulza, E. (2018). Thermal regime and host clade, rather than geography, drive Symbiodinium and bacterial assemblages in the scleractinian coral Pocillopora damicornis sensu lato. Microbiome, 6, 39. https://doi.org/10.1186/s40168-018-0423-6

Callahan, B. J., McMurdie, P. J., Rosen, M. J., Han, A. W., Johnson, A. J. A., \& Holmes, S. P. (2016). DADA2: High-resolution sample inference from Illumina amplicon data. Nature Methods, 13, 581-583. https://doi.org/10.1038/nmeth.3869

Cantin, N. E., van Oppen, M. J. H., Willis, B. L., Mieog, J. C., \& Negri, A. P. (2009). Juvenile corals can acquire more carbon from high-performance algal symbionts. Coral Reefs, 28 , 405-414. https://doi.org/10.1007/s00338-009-0478-8

Clark, J. S., Nemergut, D., Seyednasrollah, B., Turner, P. J., \& Zhang, S. (2017). Generalized joint attribute modeling for biodiversity analysis: Median-zero, multivariate, multifarious data. Ecological Monographs, 87(1), 34-56. https://doi.org/10.1002/ecm.1241

Cunning, R., Gates, R. D., \& Edmunds, P. J. (2017). Using high-throughput sequencing of ITS2 to describe Symbiodinium metacommunities in St. John, US Virgin Islands. PeerJ, 5, e3472. https://doi.org/10.7717/peerj.3472

De Cáceres, M., \& Legendre, P. (2009). Associations between species and groups of sites: indices and statistical inference. Ecology, 90(12), 3566-3574. Retrieved from http://adn.biol.umontreal.ca/ numericalecology/Reprints/De_Caceres_\&_Legendre_Ecolog y_2009.pdf

Fox, J. W. (2005). Interpreting the "selection effect" of biodiversity on ecosystem function. Ecology Letters, 8, 846-856. https://doi.org/10.1111/j.1461-0248.2005.00795.x

Frøslev, T. G., Kjøller, R., Bruun, H. H., Ejrnæs, R., Brunbjerg, A. K., Pietroni, C., \& Hansen, A. J. (2017). Algorithm for post-clustering curation of DNA amplicon data yields reliable biodiversity estimates. Nature Communications, 8(1). https://doi.org/10.1038/s41467-01701312-x 
664

665

666

667

668

669

670

671

672

673

674

675

676

677

678

679

680

681

682

683

684

685

686

687

688

689

690

691

692

693

Glasl, B., Smith, C. E., Bourne, D. G., \& Webster, N. S. (2019). Disentangling the effect of hostgenotype and environment on the microbiome of the coral Acropora tenuis. PeerJ, 7 , e6377. https://doi.org/10.7717/peerj.6377

Green, E. A., Davies, S. W., Matz, M. V., \& Medina, M. (2014). Quantifying cryptic Symbiodinium diversity within Orbicella faveolata and Orbicella franksi at the Flower Garden Banks, Gulf of Mexico. PeerJ, 2, e386. https://doi.org/10.7717/peerj.386

Halfvarson, J., Brislawn, C. J., Lamendella, R., Vázquez-Baeza, Y., Walters, W. A., Bramer, L. M., ... Jansson, J. K. (2017). Dynamics of the human gut microbiome in inflammatory bowel disease. Nature Microbiology, 2, 17004. https://doi.org/10.1038/nmicrobiol.2017.4

Hauff, B., Cervino, J. M., Haslun, J. A., Krucher, N., Wier, A. M., Mannix, A. L., .. Strychar, K. B. (2014). Genetically divergent Symbiodinium sp. display distinct molecular responses to pathogenic Vibrio and thermal stress. Diseases of Aquatic Organisms, 112, 149-159. https://doi.org/10.3354/dao02802

Howells, E. J., Berkelmans, R., van Oppen, M. J. H., Willis, B. L., \& Bay, L. K. (2013).

Historical thermal regimes define limits to coral acclimatization. Ecology, 94(5), 10781088 .

Hughes, T. P., Kerry, J. T., Álvarez-Noriega, M., Álvarez-Romero, J. G., Anderson, K. D., Baird, A. H., ... Wilson, S. K. (2017). Global warming and recurrent mass bleaching of corals. Nature, 543, 373-377. https://doi.org/10.1038/nature21707

Hughes, T. P., Kerry, J. T., Baird, A. H., Connolly, S. R., Dietzel, A., Eakin, C. M., ... Torda, G. (2018). Global warming transforms coral reef assemblages. Nature, 556, 492-496. https://doi.org/10.1038/s41586-018-0041-2

Hume, B. C. C., Ziegler, M., Poulain, J., Pochon, X., Romac, S., Boissin, E., ... Voolstra, C. R. (2018). An improved primer set and amplification protocol with increased specificity and sensitivity targeting the Symbiodinium ITS2 region. PeerJ, 6, e4816. https://doi.org/10.7717/peerj.4816

Jones, A., \& Berkelmans, R. (2010). Potential costs of acclimatization to a warmer climate: growth of a reef coral with heat tolerant vs. sensitive symbiont types. PLOS ONE, 5(5), e10437. https://doi.org/10.1371/journal.pone.0010437

Jones, A. M., Berkelmans, R., van Oppen, M. J. H., Mieog, J. C., \& Sinclair, W. (2008). A 
community change in the algal endosymbionts of a scleractinian coral following a natural bleaching event: Field evidence of acclimatization. Proceedings of the Royal Society B: Biological Sciences, 275, 1359-1365. https://doi.org/10.1098/rspb.2008.0069

Kaniewska, P., Campbell, P. R., Kline, D. I., Rodriguez-Lanetty, M., Miller, D. J., Dove, S., \& Hoegh-Guldberg, O. (2012). Major cellular and physiological impacts of ocean acidification on a reef building coral. PLoS ONE, 7(4), e34659. https://doi.org/10.1371/journal.pone.0034659

Kenkel, C. D., \& Bay, L. K. (2018). Exploring mechanisms that affect coral cooperation: symbiont transmission mode, cell density and community composition. PeerJ, 6, e6047. https://doi.org/10.7717/peerj.6047

LaJeunesse, T. C., Bhagooli, R., Hidaka, M., DeVantier, L., Done, T., Schmidt, G. W., ... Hoegh-Guldberg, O. (2004). Closely related Symbiodinium spp. differ in relative dominance in coral reef host communities across environmental, latitudinal and biogeographic gradients. Marine Ecology Progress Series, 284, 147-161. https://doi.org/10.3354/meps 284147

LaJeunesse, T. C., Smith, R. T., Finney, J., \& Oxenford, H. (2009). Outbreak and persistence of

LaJeunesse, T. C., Pettay, D. T., Sampayo, E. M., Phongsuwan, N., Brown, B., Obura, D. O., ... Fitt, W. K. (2010). Long-standing environmental conditions, geographic isolation and host-symbiont specificity influence the relative ecological dominance and genetic diversification of coral endosymbionts in the genus Symbiodinium. Journal of Biogeography, 37, 785-800. https://doi.org/10.1111/j.1365-2699.2010.02273.x 
Little, A. F., van Oppen, M. J. H., \& Willis, B. L. (2004). Flexibility in Algal Endosymbioses Shapes Growth in Reef Corals. Science, 304(5676), 1492-1495. https://doi.org/10.1126/science.1095733

Loreau, M., Naeem, S., Inchausti, P., Bengtsson, J., Grime, J. P., Hector, A., ... Wardle, D. A. (2001). Biodiversity and Ecosystem Functioning : Current Knowledge and Future Challenges. Science, 294(5543), 804-809. https://doi.org/10.1126/science.1064088

Lundgren, P., Vera, J. C., Peplow, L., Manel, S., \& van Oppen, M. J. H. (2013). Genotype environment correlations in corals from the Great Barrier Reef. BMC Genetics, 14, 9. https://doi.org/10.1186/1471-2156-14-9

Manzello, D. P., Matz, M. V., Enochs, I. C., Valentino, L., Carlton, R. D., Kolodziej, G., ... Jankulak, M. (2018). Role of host genetics and heat tolerant algal symbionts in sustaining populations of the endangered coral Orbicella faveolata in the Florida Keys with ocean warming. Global Change Biology, 25, 1016-1031. https://doi.org/10.1111/gcb.14545

Mieog, J. C., Olsen, J. L., Berkelmans, R., Bleuler-Martinez, S. A., Willis, B. L., \& van Oppen, M. J. H. (2009). The roles and interactions of symbiont, host and environment in defining coral fitness. PLoS ONE, 4(7), e6364. https://doi.org/10.1371/journal.pone.0006364

Moeller, A. H., Shilts, M., Li, Y., Rudicell, R. S., Lonsdorf, E. V., Pusey, A. E., ... Ochman, H. (2013). Siv-induced instability of the chimpanzee gut microbiome. Cell Host and Microbe, 14, 340-345. https://doi.org/10.1016/j.chom.2013.08.005

Putnam, H. M., Mayfield, A. B., Fan, T. Y., Chen, C. S., \& Gates, R. D. (2013). The physiological and molecular responses of larvae from the reef-building coral Pocillopora damicornis exposed to near-future increases in temperature and pCO2. Marine Biology, 160, 2157-2173. https://doi.org/10.1007/s00227-012-2129-9

Putnam, H. M., Stat, M., Pochon, X., \& Gates, R. D. (2012). Endosymbiotic flexibility associates with environmental sensitivity in scleractinian corals. Proceedings of the Royal Society B: Biological Sciences, 279, 4352-4361. https://doi.org/10.1098/rspb.2012.1454

Quigley, K. M., Bay, L. K., \& Willis, B. L. (2017). Temperature and Water Quality-Related Patterns in Sediment-Associated Symbiodinium Communities Impact Symbiont Uptake and Fitness of Juveniles in the Genus Acropora. Frontiers in Marine Science, 4, 401. https://doi.org/10.3389/fmars.2017.00401 
Quigley, K. M., Davies, S. W., Kenkel, C. D., Willis, B. L., Matz, M. V., \& Bay, L. K. (2014). Deep-sequencing method for quantifying background abundances of Symbiodinium types: Exploring the rare Symbiodinium biosphere in reef-building corals. PLoS ONE, 9(4), e94297. https://doi.org/10.1371/journal.pone.0094297

Quigley, K. M., Warner, P. A., Bay, L. K., \& Willis, B. L. (2018). Unexpected mixed-mode transmission and moderate genetic regulation of Symbiodinium communities in a brooding coral. Heredity, 121, 524-536. https://doi.org/10.1038/s41437-018-0059-0

Quigley, K. M., Willis, B. L., \& Bay, L. K. (2016). Maternal effects and Symbiodinium community composition drive differential patterns in juvenile survival in the coral Acropora tenuis. Royal Society Open Science, 3(10), 160471. https://doi.org/10.1098/rsos.160471

Quigley, K. M., Willis, B. L., \& Bay, L. K. (2017). Heritability of the Symbiodinium community in vertically- and horizontally-transmitting broadcast spawning corals. Scientific Reports, 7, 8219. https://doi.org/10.1038/s41598-017-08179-4

Rouzé, H., Lecellier, G., Saulnier, D., \& Berteaux-Lecellier, V. (2016). Symbiodinium clades A and D differentially predispose Acropora cytherea to disease and Vibrio spp. colonization. Ecology and Evolution, 6(2), 560-572. https://doi.org/10.1002/ece3.1895

Silverstein, R. N., Cunning, R., \& Baker, A. C. (2015). Change in algal symbiont communities after bleaching, not prior heat exposure, increases heat tolerance of reef corals. Global Change Biology, 21, 236-249. https://doi.org/10.1111/gcb.12706

Silverstein, R. N., Cunning, R., \& Baker, A. C. (2017). Tenacious D: Symbiodinium in clade D remain in reef corals at both high and low temperature extremes despite impairment. The Journal of Experimental Biology, 220, 1192-1196. https://doi.org/10.1242/jeb.148239

Smith, R. T., Pinzón, J. H., \& Lajeunesse, T. C. (2009). Symbiodinium (dinophyta) diversity and stability in aquarium corals. Journal of Phycology, 45, 1030-1036. https://doi.org/10.1111/j.1529-8817.2009.00730.x

Stimson, J., \& Kinzie, R. A. (1991). The temporal pattern and rate of release of zooxanthellae from the reef coral Pocillopora damicornis (Linnaeus) under nitrogen-enrichment and control conditions. Journal of Experimental Marine Biology and Ecology, 153, 63-74. https://doi.org/10.1016/S0022-0981(05)80006-1

van Oppen, M. J. H., Bongaerts, P., Frade, P., Peplow, L. M., Boyd, S. E., Nim, H. T., \& Bay, L. 
K. (2018). Adaptation to reef habitats through selection on the coral animal and its associated microbiome. Molecular Ecology, 27, 2956-2971. https://doi.org/10.1111/mec.14763

788

789

790

791

792

793

794

795

796

797

798

799

800

801

802

803

804

805

806

807

808

809

810

811

812

813

Witman, J. D., Etter, R. J., \& Smith, F. (2004). The relationship between regional and local species diversity in marine benthic communities: A global perspective. Proceedings of the National Academy of Sciences, 101(44), 15664-15669. https://doi.org/10.1073/pnas.0404300101

Wu, J., Peters, B. A., Dominianni, C., Zhang, Y., Pei, Z., Yang, L., ... Ahn, J. (2016). Cigarette smoking and the oral microbiome in a large study of American adults. ISME Journal, 10, 2435-2446. https://doi.org/10.1038/ismej.2016.37

Zaneveld, J. R., Burkepile, D. E., Shantz, A. A., Pritchard, C. E., McMinds, R., Payet, J. P., ... Thurber, R. V. (2016). Overfishing and nutrient pollution interact with temperature to disrupt coral reefs down to microbial scales. Nature Communications, 7, 11833. https://doi.org/10.1038/ncomms 11833

Zaneveld, J. R., McMinds, R., \& Thurber, R. V. (2017). Stress and stability: Applying the Anna Karenina principle to animal microbiomes. Nature Microbiology, 2, 17121. https://doi.org/10.1038/nmicrobiol.2017.121

Ziegler, M., Arif, C., Burt, J. A., Dobretsov, S., Roder, C., LaJeunesse, T. C., \& Voolstra, C. R. (2017). Biogeography and molecular diversity of coral symbionts in the genus Symbiodinium around the Arabian Peninsula. Journal of Biogeography, 44(3), 674-686. https://doi.org/10.1111/jbi.12913

Ziegler, M., Eguíluz, V. M., Duarte, C. M., \& Voolstra, C. R. (2018). Rare symbionts may contribute to the resilience of coral-algal assemblages. ISME Journal, 12, 161-172. https://doi.org/10.1038/ismej.2017.151

Ziegler, M., Stone, E., Colman, D., Takacs-Vesbach, C., \& Shepherd, U. (2018). Patterns of Symbiodinium (Dinophyceae) diversity and assemblages among diverse hosts and the coral reef environment of Lizard Island, Australia. Journal of Phycology, 54(4), 447-460. https://doi.org/10.1111/jpy.12749 